\title{
Cooperation Mechanism Taxonomy for Wireless Sensor and Actor Networks
}

\author{
Erica Ruiz-Ibarra, Luis Villasenor-Gonzalez \\ \{cruiz, luisvi\}@cicese.mx \\ CICESE Research Center, Department of Electronics and Telecommunications \\ Km. 107 Carretera Tijuana-Ensenada, Ensenada, México 22860
}

\begin{abstract}
A Wireless Sensor and Actor Network (WSAN) is composed of sensor and actor nodes distributed in a geographic area of interest; the sensors are involved in monitoring the physical environment, while the actors can execute a designated task in accordance to the data collected and reported by the sensors during an event. To achieve a balanced performance, a WSAN architecture must implement an efficient cooperative communication strategy to allow the nodes to collaborate in the optimal assignment of resources and to execute tasks with the lowest possible delay. Such collaboration must take place by exchanging information and generating negotiated decisions while trying to extend the WSAN lifetime. The main contribution of this work is the proposal of a coordination mechanism taxonomy for WSANs; this taxonomy provides a framework for the classification of coordination mechanisms designed for WSAN environments. Based on this taxonomy, a comparative analysis is presented to study some of the most representative coordination mechanisms proposed in the area of WSANs up to this date.
\end{abstract}

Keywords: Coordination mechanism, Wireless sensor and actor networks, WSN, WSAN, Taxonomy.

\section{Introduction}

Recent advances in microelectronics and wireless technology have enabled the development of small size devices, which are low cost, power limited and equipped with wireless communication capabilities. This has led to the development of Wireless Sensor Networks (WSN), which are composed of hundreds of sensor nodes used to monitor multiple physical variables, such as temperature, humidity, sound, pressure, movement, vibrations, etc. [1]. A WSN can be deployed to support a large number of applications which include environmental monitoring, inventory tracking, prediction of natural disasters (e.g. earthquakes, forest fires), home automation, traffic control and military supervision in the battlefield [1]. However, there are some complex scenarios that require the cooperation between sensors and higher capability devices, such as actor nodes, to support the proper execution of specific tasks; wireless sensor and actor networks (WSAN) have been proposed as an important extension of WSN [2]. A WSAN can be deployed for a great variety of applications, such as microclimate control in a building or a greenhouse, detection of biological, chemical or nuclear attacks, automation of industrial processes, control of ventilation systems and heating [3].

Please use the following format when citing this chapter:

Ruiz-Ibarra, E. and Villasenor-Gonzalez, L., 2008, in IFIP International Federation for Information Processing, Volume 264; Wireless Sensor and Actor Networks II; Ali Miri; (Boston: Springer), pp. 62-73. 
A WSAN is composed of a large amount of sensor nodes and a few actors connected by wireless means; these devices cooperate among themselves to provide distributed sensing and to execute specific tasks [4]. Sensor and actor nodes can be spread in the field, while a sink node can be used to monitor the network and may be used to communicate with a task manager, as illustrated in Figure 1. In a WSAN the sensor nodes behave as passive elements, collecting information from the physical world, whereas the actors are active elements that make independent decisions and are capable of executing appropriate actions in accordance to the information collected; all these capabilities allow the user to monitor and to act when located in a remote location [5]. In a WSN scenario, it is usually assumed that sensor nodes cannot be locally configured or recharged while deployed in the field, therefore this type of devices are required to be autonomous and energy efficient. The energy constraint of the sensor nodes impose limitations on the size of the device, similarly, there is a reduction of resources like memory, processing speed, computing power and bandwidth [1]; all of this with the purpose of extending the lifetime of the sensor node. On the other hand, in a WSAN, the actor nodes are equipped with greater resources, such as increased computing capacity, powerful transmitters and increased battery lifetime by means of rechargeable or replaceable power sources [5].

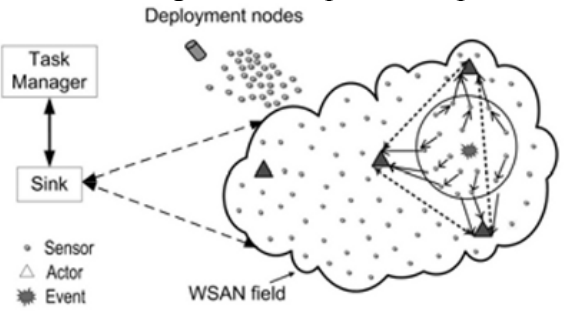

Fig. 1 Physical architecture of a WSAN [5]

The main objective of this work is to provide a common framework for the study and analysis of cooperative mechanisms in a WSAN environment. The remainder of this paper is organized as follows. Section 2 presents a description of the WSAN network architecture. Section 3 outlines a novel taxonomy for the study and the analysis of coordination mechanisms in a WSAN environment. Section 4 provides a classification and a comparative analysis of the WSAN coordination mechanisms by employing the taxonomy introduced in section 3. Finally section 5 states the conclusions of this work.

\section{WSAN Architecture}

A WSAN is a distributed system that can adapt and react to the environmental conditions which are reported by the collaborative effort of all the sensors and actors $[6,7]$. Two different types of architectures can be defined according to the way data is collected by the sensor nodes and is reported back to the actor nodes; these are defined as the Automated and Semi-automated architectures, illustrated in Figure 2. In the Automated architecture, data is collected by the sensor nodes and it is transmitted 
directly to the actors, which will efficiently coordinate to execute a specific task without collaboration from the sink. As a result, automated WSAN architectures are recommended for time sensitive applications where a fast reaction by the actors is a critical requirement. In the Semi-automated architecture, the sensor data is transmitted to a central controller (e.g. the sink) which will process the collected data and will determine which actors must take action to execute a specific task; this is accomplished by transmitting a set of commands to the corresponding actors.

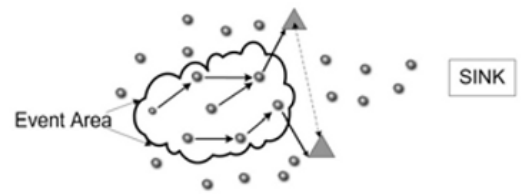

a)

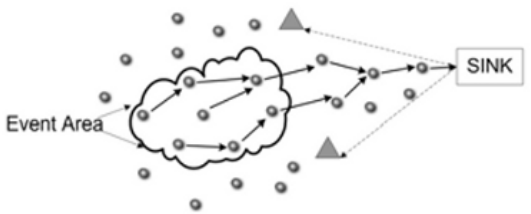

b)

Fig. 2 a) Automated and b) Semi-automated Architecture [5]

The communication process, in a WSN environment, mainly takes place from the sensor nodes to the sink; on the contrary, the communication process in a WSAN environment can take place between the sensor and the actor nodes. Thus, a WSAN architecture requires the implementation of multiple coordination levels; these coordination levels are defined as: Sensor-Sensor (SS), Sensor-Actor (SA) and ActorActor (AA). The SS coordination is employed to gather information from the physical world in an effective and energy efficient way. The SA coordination is employed to report new events and to transmit the characteristics of the event from the sensors to the actors [7]; in addition, the SA coordination may also be used over the downlink (i.e. from the actor toward the sensor) to inform the sensors to proceed with specific sensing tasks. The AA coordination is required to execute a specific task while coordinating which actor nodes should respond within a certain area. The objective of these mechanisms is to coordinate the actions between the sensor and the actor nodes, while making an optimum use of the available resources and at the same time executing the required tasks within the time bound required by the application [8].

The following section presents a coordination mechanism taxonomy. This taxonomy is proposed to provide a framework for the analysis and evaluation of coordination mechanisms used in WSAN architectures.

\section{A Taxonomy for WSAN Coordination Mechanism}

To the best of our knowledge, there is no specific coordination mechanism taxonomy for WSAN networks. There is some related work, like Farinelli's [9], which presents a taxonomy for the coordination of multi-robot systems and is based in four levels: cooperation, knowledge, coordination and organization. However, this taxonomy only considers cooperative systems consisting of robots and a coordination protocolbased/protocol-free decision making process, but it does not include data and context sharing. Another related work by Salkhman et al. [10] presents a taxonomy for 
context aware collaborative systems based on the commonalities of different contextaware systems that emphasize collaboration, however this taxonomy is too broad, as it can be applied to a great variety of systems, from small augmented artifacts to large scale and highly distributed sensor/(actor) networks; as a result, the proposed structure does not provide the proper elements for the fine classification required in a WSAN coordination mechanism. The structure proposed by Salkhman is supported in three axis: Goal, Approaches and Means. A third work by Sameer et al. [11] develops a range of middleware services such as synchronization, localization, aggregation and tracking to facilitate the coordination through self-organizing networked sensor.

A new taxonomy for WSAN coordination mechanisms is proposed in this section; this proposal is inspired from the work presented by Salkhman [10] and Sameer [11]. The proposed taxonomy is divided in four sections as shows in Figure 3.

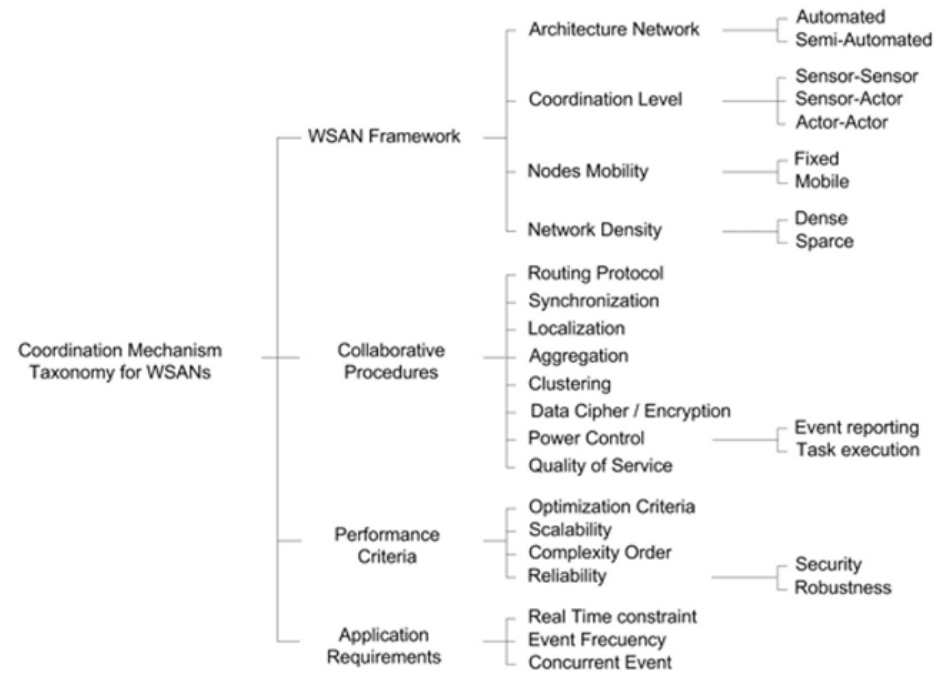

Fig. 3 Coordination Mechanism Taxonomy for WSANs

WSAN Framework. It represents the structure of a WSAN and includes:

- Network architecture. It is related to the way information is sensed and reported to the actors, which may be automated or semi-automated.

- Coordination Levels. It refers to the coordination levels employed by the coordination mechanism.

- Node mobility. It is used to specify if the nodes in a WSAN (i.e. sensors, actors or the sink) are mobile or fixed.

- Network density. It defines the ratio between the number of nodes spread throughout the field and the field dimensions.

Collaborative Procedures. It refers to those collaborative procedures between the elements of a WSAN, used to support the exchange of information by the coordination mechanism; these services include:

- Routing Mechanism. It is related to the procedure implemented for selecting a route to transmit packets to a destination. 
- Synchronization. It refers to the implementation of energy-efficient techniques required to associate time and location information with the sensed data.

- Aggregation. It has the objective of reducing the energy consumption and the control overhead associated with the transmission of information to a common destination. To achieve this goal, a single packet is created as a result of the fusion of data which is generated by multiple sources.

- Localization. It is implemented to provide information regarding the geographical location of the sensor and actor nodes.

- Clustering. It provides a hierarchy among nodes; the substructures that are collapsed in higher levels are called cluster and there is at least one node in each cluster which is denoted as the cluster-head.

- Power Control. It is related to the possibility of varying the transmission power; as a result, the radio signal coverage of a node can be modified.

- Quality of Service $(Q o S)$. It is related to the mechanisms employed to provide guaranteed services in a WSAN architecture. In a WSAN scenario, it is possible to define two different functionalities to provide QoS support. One functionality is based on providing the required resource reservations to the nodes involved in reporting an event at the SA coordination level; in this way, it will be possible to provide differentiated services to the data being transmitted in the WSAN. A different functionality is to prioritize the execution of tasks by the actors in response to events in the WSAN. Thus, the actors should be able to respond in accordance to the priority of the events reported by the sensor nodes.

- Data Cipher and/or Encryption. It is related to the implementation of any cipher/encryption mechanism used to protect the integrity of data in the WSAN.

Performance Criteria. It is related to those criteria elements used to estimate the performance of the coordination mechanism; such as:

- Optimization parameters. This criteria denotes those metrics in which the mechanism is based to reach the proposed objectives.

- Complexity Order. It provides a measurement of the computational complexity of the proposed algorithms.

- Reliability. It provides a measure of the level of security and robustness of a WSAN coordination mechanism. To classify a coordination mechanism as secure, it must implement additional functionalities to guarantee data integrity and to avoid access to the WSAN by intruders. On the other hand, robustness is related to the capability of the coordination mechanism to handle faults, while assuring data delivery from the sensors to the actors by means of acknowledgement or retransmission procedures.

Application Requirements. The coordination mechanism must take into consideration the application requirements, such as:

- Real-Time constraints. It is related to the amount of time required by the coordination mechanism to report events and execute a task.

- Event Frequency. It is related to the periodicity of events at which the system is capable of providing a proper response.

- Concurrent Event Support. It is related to the capability of the coordination mechanism to support multiple simultaneous events in a WSAN. 


\section{Comparative Analysis}

This section presents a comparison of the most relevant coordination mechanisms published up to this date in the literature. This comparison is based on the coordination mechanism taxonomy introduced in section 3; this taxonomy is divided in four sections and Tables 1- 4 show a summary of the comparative analysis for each of these. The coordination mechanism proposals are referenced using the following notation: the work presented by Melodia et al. in $[12,13]$ is referenced as $A$; the framework proposed by Ngai et al. [14] is referenced as $B$; the coordination mechanism provided by Yuan et al. [15] is referenced as $C$; the proposal presented by Shah et al. [16] is referenced as $D$; and the architecture developed by Melodia et al. [17] is referenced as $E$.

WSAN Framework. It represents the structure of a WSAN. The Table 1 shows the comparative analysis for the WSAN Framework.

Table 1 WSAN Framework comparison

\begin{tabular}{|c|c|c|c|c|c|}
\hline \multirow[b]{2}{*}{$\begin{array}{l}\text { WSAN Framework } \\
\text { Networks Architecture }\end{array}$} & \multicolumn{5}{|c|}{ Coordination Mechanism Approach } \\
\hline & $\mathbf{A}[12,13]$ & B [14] & $\mathrm{C}[15]$ & D [16] & $\mathbf{E}[17]$ \\
\hline Automated & Yes & Yes & Yes & Yes & Yes \\
\hline Semi-Automated & No & No & No & Yes & No \\
\hline Coordination Level & & & & & \\
\hline Sensor-Sensor & No & Yes & Yes & Yes & No \\
\hline Sensor-Actor & Yes & Yes & Yes & Yes & Yes \\
\hline Actor-Actor & Yes & Yes & Yes & No & Yes \\
\hline Node Mobility & & & & & \\
\hline Sensor & Fixed & Fixed & Fixed & Fixed & Fixed \\
\hline $\begin{array}{l}\text { Actor } \\
\text { Sink }\end{array}$ & Fixed & Mobile & Mobile & $\begin{array}{l}\text { Mobile } \\
\text { Fixed }\end{array}$ & Mobile \\
\hline Network Density & $\begin{array}{l}\text { Dense } \\
\text { Sparse }\end{array}$ & Dense & Dense & Dense & Dense \\
\hline
\end{tabular}

WSAN Architecture. From Table 1, it can be seen that all of the coordination mechanisms support the automated architecture given its low latency; due to the direct communication between sensors and actors. In proposal $\mathrm{D}$, the authors present a coordination mechanism and evaluate it using both the automated and semiautomated architectures.

Supported Coordination Levels The coordination levels supported by each of the proposed mechanisms differ. In $A$, the SS coordination functions do not take place, as the sensors proceed to associate with an actor immediately after the detection of an event; in this way the sensor nodes will form a cluster with the actor as the clusterhead. In $B$ the architecture implements clustering and data aggregation techniques between sensors, and the data corresponding to each cluster is reported to the closest actor; this is defined as SS and SA event notification, which evidently imply SS and SA coordination functions. In $C$, the proposed coordination mechanism supports the 
implementation of the SS, SA, and AA coordination levels. In $D$, the sensors are grouped into clusters and the average packet delay is estimated via the DAWC protocol, all these tasks are related with the SS coordination. In relation to proposal $E$, the coordination mechanism operates at the SS and AA coordination levels; it should be noticed that proposal $E$ considers mobile actors and was developed as an extension of proposal $A$.

Node Mobility. The proposals summarized in Table 1, assume that the sensor nodes are fixed, while most of the proposals assume the actors to be mobile, with the exception of $A$ which assumes the actors to be fixed. By considering mobile actors it is possible to consider a broader range of applications of a WSAN architecture; all this at the expense of increased complexity, as the actor mobility requires an efficient coordination.

Network Density. In relation to the network density all the proposals are capable of operating in a high density topology; however, some of the proposals do not explicitly specify this capability, as in $B$. Nonetheless it is assumed that $B$ is scalable as it relies on cluster formation and data aggregation during the reporting of an event. Regarding proposal $D$, the authors explicitly describe that it can manage dense, as well as, sparse deployment of nodes.

Collaborative Procedures. Table 2 summarizes the collaborative procedures implemented in proposals $A$ through $E$.

Table 2 Collaborative Procedures

\begin{tabular}{lccccc}
\hline \multicolumn{1}{c}{ Collaborative } & \multicolumn{4}{c}{ Coordination Mechanism Approach } & \\
Procedures & A [12, 13] & B [14] & C [15] & D [16] & E [17] \\
Routing Protocol & Geographical & Geographical & GAF (SS) & C-DEAR & Geographical \\
& & & Ad-hoc (AA) & D-DEAR & \\
Synchronization & Yes & & Yes & Yes & Probably \\
Localization & Yes & Yes & Yes & Yes & Yes \\
Aggregation & Yes & Yes & Yes & NS & NS \\
Clustering & Event-driven & Event-driven & Hierarchical & Dynamic & \\
& & & Geographical & Weighted & \\
Power Control & Yes & No & No & No & Yes \\
Cipher/Encryption & No & No & No & No & No \\
QoS & & & & & \\
Event reporting & Yes & No & No & No & Yes \\
Task execution & No & No & Yes & No & Yes \\
\hline
\end{tabular}

Routing Support. Most of the proposed coordination mechanisms make use of geographical routing protocols, given their scalability and the fact that they can easily adapt to the location changes of the actor nodes $[13,18,19]$; in addition, it is possible to exploit the localization information of the nodes to route packets to the intended nodes in accordance with their localization within an event area. In $A, B$ and $E$ the SA coordination is based on a geographical routing paradigm, while the proposal presented in $C$ makes use of the GAF [20] (Geographical Adaptive Fidelity) routing protocol at the first coordination level; in this way the sensor nodes can use the geographical localization information to transmit the data to the cluster-head, which will in turn forward the data to the closest actor. In addition, $C$ proposes the use of 
conventional Ad Hoc routing protocol at the last level (i.e. AA coordination level), given the reduced number of actor nodes and their higher performance capabilities. On the other hand, $D$ proposes the implementation of Delay and Energy Aware Routing protocols, such as the DEAR protocol, to transmit the data to the sink or to the actors. In the semi-automated scenario, $D$ proposes the implementation of a centralized DEAR (C-DEAR) protocol, while for automated architectures it implements a distributed DEAR (D-DEAR) protocol; these two implementations of the DEAR protocol are designed to comply with the end-to-end delay requirements for real-time applications. In proposal $E$, the authors derive a simple yet optimal forwarding rule based on geographic position in presence of Rayleigh fading channels.

Synchronization. The synchronization technique is only implemented in proposals $A, C$ and $D$; proposals $B$ and $E$ do not make any reference to the requirement of a time synchronization mechanism. In $A$, it is assumed that the network is synchronized by means of the implementation of an existing synchronization protocol. In $C$ the actors will periodically transmit their geographical coordinates along with a timestamp; this information allows the cluster-head to synchronize with the actors. In $D$, a time synchronization mechanism is assumed to be implemented, as packet delay measurements are made by the receiver which implies that packets are tagged with timestamp information. With respect to proposal $E$, it does not explicitly make a reference to the requirement of a synchronization technique; however it is assumed that a time synchronization service is required, as the location estimation of the actors is made during specific time intervals.

Node Localization. The localization service is considered to be implemented by all the proposals compared. It is assumed that all the nodes are capable of knowing or determining their geographical localization; this can be implemented by means of a Global Positioning System (GPS) receiver, through trilateration techniques or any other similar approach. In proposal $E$, the authors make reference to a hybrid location management scheme to handle the mobility of actors with minimal energy expenditure, based on update messages sent by mobile actors to sensors.

Aggregation. The data fusion service is implemented in $A, B$ and $C$, while $D$ and $E$ does not specify it. In $A$, data fusion is only implemented at the SA coordination level whenever a sensor receives information from at least two other sensors; the data is then relayed toward the actor node. In $B$ the data fusion is implemented at the SS coordination level and further divided into different layers according to their importance; the aggregated data is then transmitted to the closet actor in the order of significance. In $C$ data fusion is implemented at the three coordination levels, that is, the cluster-head will perform data fusion on the data received from the member nodes (i.e. fusion at the first level); at the SA coordination level all the cluster-heads associated to the same actor will construct a data-aggregation tree toward the actor; and finally at the AA coordination level, all the actors activated by a common event will construct a third data-aggregation tree toward the actor located at the center of the event area.

Clustering. The cluster formation approach is implemented in proposals $A, B, C$ and $D$; on the contrary, proposal $E$ does not rely on a cluster formation mechanism. In relation to proposals $A$ and $B$, they both implement the event-driven clustering paradigm. In the event-driven clustering, the cluster formation process is triggered by 
an event and the clusters are created on-the-fly. Proposal $C$ is based on a hierarchical geographical clustering paradigm, where the cluster formation is done by splitting the action area in smaller sections to create virtual grids; this strategy reduces the traffic load within each grid and makes an efficient use of resources. Finally, $D$ proposes a Dynamic Weighted Clustering Algorithm (DAWC) which adapts to the dynamic topology of the networks; the procedure of cluster formation is not periodic and it is based on a weighting equation which sets weights to different parameters according to the application needs.

Power control. A power control mechanism is implemented in proposals $A$ and $E$. Proposal $A$, employs a power control mechanism in the actors, which can select their power among L different levels. A higher power corresponds to a lower action completion time. On the other hand, proposal $E$ employs a power control mechanism at the SA coordination level, where the sensors can increase the forwarding range in order to adjust the end-to end delay.

Data Cipher and/or Encryption. None of the coordination mechanisms compared make use of a security mechanism, such as ciphering or encryption, to protect the integrity of data. As a result, there is an open opportunity area for the development of coordination mechanisms which may incorporate ciphering and or encryption techniques.

Quality of Service $(Q o S)$. In relation to Data Transfer Priorities the proposal $A, D$ and $E$ implement a data transfer priority scheme. Proposal $A$ introduces a novel notion of "reliability", which is defined as the minimum latency required by the application. To provide the required reliability, with minimum energy expenditure, $A$ proposes a Distributed Event-driven Partitioning and Routing (DEPR) protocol, as explain in [12]. Proposal $D$ relies on a clustering algorithm DAWC which estimates the delay budget for forwarding a packet from the cluster-heads; this algorithm guarantees the packet delivery delay to be within the given delay bound. Proposal $E$, similarly to $A$, uses the concept of "reliability", but in this case the application "reliability" requirement is achieved by adjusting the end-to-end delay by means of a power control mechanism when the traffic generated in the event area is low, and by means of a actor-driven congestion control scheme in case of congestion. Proposals $C$, and $B$ are concerned with reducing the latency during the event reporting stage, during the transmission of event related information from the sensors toward the actors, but do not implement a priority scheme to support different application requirements. Regarding the Task Assignment Priorities, proposals $C$ and $E$ make use of a task priority assignment technique. In proposal $C$, according to the characteristic of the event, one or more actors can be triggered to perform one or more task. In proposal $E$, the task assignment process is achieved by means of a Mixed Integer Non-Linear Program [21] (MINLP), where the event is characterized by a tuple that describes the event characteristics, in addition it includes an event preemption policy for multiactor task allocation for cases where resources are insufficient to accomplish a high priority task.

Performance Criteria. Table 3 shows a summary of the comparative analysis for the performance criteria.

Optimization Criteria. All of the proposals make use of the energy and latency as optimization metrics; in $A$ the maximum allowed latency is defined as reliability. In addition to these parameters, proposals $A$ and $E$ measure the packet loss rate. 
Scalability. Proposals $A, B, C$ and $E$ make use of a geographical routing protocol which is becoming the most promising scalable solutions for critically energyconstrained sensor networks $[18,19]$. On the other hand, proposals $A, B, C$ and $D$ make use of clustering schemes which promote scalability and an efficient use of energy in the network.

Table 3 Performance Criteria comparison

\begin{tabular}{|c|c|c|c|c|c|}
\hline Performance & \multicolumn{5}{|c|}{ Coordination Mechanism Approach } \\
\hline Criteria & $A[12,13]$ & B [14] & $\mathrm{C}[15]$ & D [16] & E [17] \\
\hline & Energy & Energy & Energy & Energy & Energy \\
\hline $\begin{array}{l}\text { Opumization } \\
\text { criteria }\end{array}$ & $\begin{array}{l}\text { Latency } \\
\text { Loss packet }\end{array}$ & Latency & Latency & Latency & $\begin{array}{l}\text { Latency } \\
\text { Lost packet }\end{array}$ \\
\hline Scalability & Yes & Yes & Yes & Yes & Yes \\
\hline $\begin{array}{l}\text { Complexity Order } \\
\text { Reliability }\end{array}$ & - & - & - & - & - \\
\hline Security & No & No & No & No & No \\
\hline Robustness & No & No & No & No & No \\
\hline
\end{tabular}

Complexity Order. None of the proposals provide a clear analysis regarding the memory and the computational resources required to support the proposed coordination mechanism. In relation to $D$ and $E$, they provide a measure of the order of complexity for some aspects of the coordination mechanism. However, none of the proposals provide a complete analysis to help determine the complete order of complexity of the coordination mechanism as a whole.

Reliability. It is related to the security and the robustness of the coordination mechanism. With respect to security, none of the proposals make use of a procedure to guarantee data integrity, or avoid network access to intruders. Regarding robustness, none of the proposals implement, or propose, a procedure to guarantee fault tolerance. Some proposals only measure the packet loss rate and show how the algorithms, implemented to reduce latency, also help to reduce the packet loss; however, no procedure is proposed to guarantee packet delivery through the use of acknowledgement messages or the retransmission of information.

Application Requirements. The coordination mechanism must take into consideration the application requirements. Table 4 shows a summary of the comparative analysis of the application requirements.

Table 4 Application Requirements comparison

\begin{tabular}{|c|c|c|c|c|c|}
\hline Application & \multicolumn{5}{|c|}{ Coordination Mechanism Approach } \\
\hline Requirements & $\mathrm{A}[12,13]$ & B $[14]$ & $\mathrm{C}[15]$ & D [16] & E [17] \\
\hline Real time constraint & Low Latency & Low Latency & Yes & Yes & Low Latency \\
\hline Event Frequency & Low & Low & High & High & High \\
\hline Concurrent events & No & No & Yes & Yes & Yes \\
\hline
\end{tabular}

Real-Time constraints. In general, the proposed coordination mechanisms support real-time applications, as they promote latency reduction during the event reporting process and the execution of tasks. In relation to proposals $A, B$ and $E$, they introduce 
a network configuration delay due to the event-based clustering approach they implement; nonetheless, these proposals try to reduce the event reporting latency. As a result, these proposals may not be suitable for real-time applications.

Frequency of Events and Concurrent event support. With respect to the frequency of events that can be handled by the coordination mechanism, proposals $A$ and $B$ are not capable of providing an efficient operation under high frequency event scenarios, this is also true for scenarios involving multiple events; this is related to the limitations imposed by the event-driven clustering paradigm. In relation to proposals $C, D$ and $E$, they are able to operate in frequent-event scenarios, as well as, scenarios involving multiple simultaneous events.

\section{Conclusions}

This work analyzes the different cooperation strategies implemented at the nodes of a WSAN, which are used to achieve distributed sensing and the execution of tasks in accordance to the sensed data. One of the main contributions of this work is the proposal of coordination mechanism taxonomy for WSAN architectures; the taxonomy provides a framework suited to the specific requirements of a coordination mechanism designed for a WSAN environment. The proposed taxonomy is divided in four sections: WSAN Framework, Collaborative Procedures, Performance Criteria and Application Requirements.

In addition to the taxonomy, a comparative analysis of the most representative coordination mechanism, published in the literature up to this date, has been provided. In general, the proposed mechanisms proceed to split the event area and perform a hierarchical coordination by employing localization information; this is done with the objective of selecting the proper nodes (i.e. sensors and actors) that will react in response to a specific event with the smallest possible response time. The proposed applications for each of the coordination mechanisms differ with respect to the frequency of the events. In general, the proposed coordination mechanisms try to comply with the support of real-time response requirement along with an efficient use of energy in the WSAN. On a final note, none of the coordination mechanisms analyzed implement a mechanism that guarantee the security of the data and system robustness.

\section{References}

1. Romer, Kay, Mattern F: The Design Space of Wireless Sensor Networks. IEEE Wireless Communications, (2004).

2. Vassis D, Kormentzas G, Skianis C: Performance evaluation of single and multi-channel actuator to actuator communication for wireless sensor actuator networks. Ad Hoc Networks, Elsevier Science, (2006).

3. Petriu EM, Georganas ND, Petriu DC, Makrakis D, Groza VZ: Sensor-based information appliances. IEEE Instrumentation and Measurement Magazine (2000).

4. Akyildiz Ian F, Su W, Sankarasubramaniam Y, Cayirci E: A Survey on Sensor Networks. IEEE Communications Magazine (2002). 
5. Akyldiz F, Kasimoglu I: Wireless sensor and actuator networks: Research challenges. Ad Hoc Networks Journal Elsevier. (2004).

6. Cayirci E, Coplo T, Emiroglu O: Power Aware Many to Many Routing in Wireless Sensor and Actuator Networks. Proc. 2nd European Workshop on Wireless Sensor Networks, (2005).

7. Hu F, Cao X, Kumar S, Sankar K: Trustworthiness in Wireless Sensor and Actuator Networks: Toward Low-complexity Realibility and Security. IEEE Global Telecommunications Conference, GLOBECOM, (2005).

8. Díaz M, Garrido D, Llopi L, Rubio B, Troya JM.: A component framework for wireless sensor and actuator network. 11th IEEE International Conference on Emerging Technologies and Factory Automation. (2006).

9. Farinelli A, Iocchi L, Nardi D: Multirobot systems: a classification focused on coordination. Systems, Man and Cybernetics, Part B, IEEE Transactions Volume 34. (2004).

10. Salkham A, Cunningham R, Senart A, Cahill V: A Taxonomy of Collaborative ContextAware Systems. Workshop on Ubiquitous Mobile Information and Collaboration Systems UMICS'06. (2006).

11. Sundresh S, Agha G, Mechitov K, Kim WY, Kwon YM: Coordination Services for Wireless Sensor Networks. International Workshop on Advanced Sensors, Structural Health Monitoring and Smart Structures. (2003).

12. T Melodia, D Pompili, VC Gungor, and IF Akyildiz: A distributed coordination framework for wireless sensor and actuator networks. Proc. 6th ACM international symposium on mobile ad hoc networking and computing. 2005.

13. Melodia T, Pompili D, Gungor VC, Akyildiz IF: Communication and Coordination in Wireless Sensor and Actor Networks, IEEE Transactions on Mobile Computing, (2007).

14. Ngai ECH, Lyu MR, Liu J: A Real Time Communication Framework for Wireless SensorActor Networks. IEEE Aerospace Conference, (2006).

15. Yuan H, Huadong M, Hongyu L: Coordination Mechanism in Wireless Sensor and Actuator Networks. First International Multi-Symposiums on Computer and Computational Sciences - IMSCCS'06. (2006).

16. Shah, G.A, Bozyigit M, Akan OB, Baykal B: Real Time Coordination and Routing in Wireless Sensor and Actuator Networks. Proc. 6th International Conference on Next Generation Teletraffic and Wired/Wireless Advanced Networking NEW2AN (2006).

17. Melodia T, Pompili D, Akyldiz I: A Communication Architecture for Mobile Wireless Sensor and Actor Networks. In Proceeding of IEEE International Conference on Sensor, Mesh and Ad hoc Communications and Networks SECON. (2006).

18. J Li, J Jannotti, DD Couto, D Karger, and R Morris: A scalable location service for geographic ad hoc routing, in Proceedings of ACM/IEEE MobiCom 2000, Boston, Massachussets. (2000)

19. R Jain, A Puri, and R Sengupta: Geographical routing using partial information for wireless ad hoc networks, IEEE Personal Communications. (2001)

20. Y Xu, J Heidemann, and D Estrin: Geographyinformed Energy Conservation for Ad Hoc Routing, Proceedings of the 7th annual international conference on Mobile computing and networking, (2001).

21. J Czyzyk, M Mesnier, and J More: The NEOS server, IEEE Journal on Computational Science and Engineering, vol. 5. (1998). 